\title{
Supporting the upper body with the hand on the thigh reduces back loading during lifting
}

\author{
Idsart Kingma*, Gert S. Faber, Jaap H. van Dieën \\ Research institute MOVE, Department of Human Movement Sciences, VU University Amsterdam, Amsterdam, Netherlands
}

\section{A R T I C L E I N F O}

Article history:

Accepted 14 September 2015

\section{Keywords:}

Lifting

Low-back load

Spine

Compression

One handed lifting

Biomechanics

\begin{abstract}
A B S T R A C T
When picking objects from the floor, low back pain patients often tend to support the upper body by leaning with one hand on a thigh. While this strategy may reduce back load, this has not yet been assessed, probably due to the difficulty of measuring the forces between hand and thigh.

Ten healthy male subjects lifted a pencil and a crate from the floor, with four lifting techniques (free, squat, stoop and a Weight Lifters Technique (WLT)), each of which was performed with and without supporting with one hand on the thigh. A six Degrees of Freedom force transducer, with a comfortable surface to support the hand on, was mounted just above the subject's left knee. Hand forces, ground reaction forces, full body kinematics, and trunk EMG were measured. Using inverse dynamics and taking the forces between hand and thigh into account, we calculated 3D L5S1 joint moments, and subsequently estimated spine forces using an EMG-assisted trunk model.

For lifting a pencil, hand support reduced average peak total moments by $17-25 \%$, dependent on lifting technique. For crate lifting, hand support reduced total moments by $13-19 \%$ compared with onehanded lifting and by $14-26 \%$ compared to two-handed lifting. Hand support slightly increased asymmetric motions and caused a substantial increase in asymmetric moments in crate lifting. For compression forces, reductions (up to $28 \%$ ) were seen in all techniques except in stoop lifts. It is concluded that leaning with a hand on the thigh can lead to substantial reductions of low back loading during lifting.
\end{abstract}

(c) 2015 Elsevier Ltd. All rights reserved.

\section{Introduction}

Manual lifting is associated with the risk of developing low-back pain (Hoogendoorn et al., 2000; Norman et al., 1998) and in recent work cumulative back load due to manual materials handing has been shown to be prospectively related to the development of back pain (Coenen et al., 2014; Marras et al., 2014). Indeed, spine loads during lifting (Arjmand et al., 2009; Kingma et al., 2004; Lavender et al., 2009; Marras and Davis, 1998) have been shown to be high enough to cause tissue damage in vitro (Brinckmann et al., 1989), and this risk of damage increases with repeated loading (Coenen et al., 2012).

Much research has been dedicated towards finding lifting techniques that reduce back loads (Anderson and Chaffin, 1986; Bazrgari et al., 2007; de Looze et al., 1998; Gagnon et al., 1993; Kingma et al., 2004; Kingma et al., 2006; Kingma et al., 2010; Lariviere et al., 2002; Lavender et al., 2007; Troup et al., 1983; van Dieën et al., 1999), but evidence concerning the optimal technique remains inconclusive

\footnotetext{
* Correspondence to: Department of Human Movement Sciences, VU University, Van der Boechorststraat 9, 1081 BT Amsterdam, Netherlands. Tel.: +31 205988492. E-mail address: I.KINGMA@VU.NL (I. Kingma).
}

(Burgess-Limerick, 2003; Straker, 2003; van Dieën et al., 1999). This might be due to the fact that effects of lifting technique are highly dependent on object dimensions and initial hand height (Dolan et al., 1994; Faber et al., 2009; Kingma et al., 2004; Kingma et al., 2006; Kingma et al., 2010).

For sagittal symmetrically positioned objects, one of the lifting techniques that has yet received little attention, but showed consistent evidence to date, is one-handed lifting.

Compared to two-handed lifting, lifting with one hand showed a minor (5-10\%) reduction in lumbar moments or compression forces (Kingma and van Dieën, 2004; Marras and Davis, 1998), whereas more substantial (15-30\%) reductions were found when the free hand was used to support the upper body by leaning on an external object (Ferguson et al., 2002; Kingma and van Dieën, 2004).

However, in daily life, suitable objects to lean on are often not available when objects are to be lifted from floor level. Subjects with low back pain often intuitively, or through instruction, use their free hand or elbow to lean on a thigh when they pick up an object from the floor. It is plausible that the resulting upward forces on the arm reduce back loading during lifting just like when leaning on an external object. However, probably as a result of the difficulty of assessing the support forces involved, to date, this common way of lifting has never 
been assessed. The present study therefore aims to assess the effects of supporting the upper body by leaning with one hand or elbow on the thigh. As the magnitude of support forces and their consequence for spine loading may vary with lifting technique, we assessed support effects for four lifting techniques, both for lifting a pencil and a crate from floor level. We hypothesized that hand and elbow support reduce spine loading, at the cost of some increase of asymmetric load components. In addition we hypothesized that these effects vary with lifting technique, with techniques that allow for large support forces showing the largest effects.

\section{Methods}

After signing an informed consent, 10 healthy young males (age 25.0 yrs, SD 6.9 yrs, weight $70.9 \mathrm{~kg}$, SD $8.8 \mathrm{~kg}$, height $1.77 \mathrm{~m}$, SD $0.06 \mathrm{~m}$ ) participated in the experiment. None of the participants had a history of low-back pain. The experiment was approved by the local ethics committee.

\subsection{Experimental design and procedure}

During the experiment, participants lifted a pencil from floor level and a $15 \mathrm{~kg}$ crate (height $0.247 \mathrm{~m}$, depth $0.297 \mathrm{~m}$, width $0.498 \mathrm{~m}$ ) from a shelf, suspended $50 \mathrm{~mm}$ above the surface of the force plate that the participants were standing on.
Both the crate and the pencil were placed at in the mid-sagittal plane. For both the pencil and the crate 24 lifts were performed, 2 repetitions of four lifting techniques at three support conditions. For the pencil, support conditions were no support, supporting the upper body by once leaning with the elbow and once leaning with the hand of the free arm on a support surface on top of a force transducer mounted just above the knee. For hand support, subjects were instructed to lean with the palm of their left hand on the support surface, and not exert force with their fingers on their knee. The crate contained three handles, two at each side, and one at the center, all at a height of $0.215 \mathrm{~m}$ in the crate. Support conditions for the crate were: lifting with two hands at handles on the side of the crate, and lifting with one hand using the handle at the center of the crate, once while the free hand was not used, and once while the free left hand was used to support the upper body by leaning on the force transducer. Note that we did not include an elbow support condition for crate lifting as pilot work showed that most subjects would not naturally reach close to the knee with the elbow when reaching for the crate handle. The force transducer was a six degrees of freedom transducer (ATI mini45 SI-580-20; $15.7 \mathrm{~mm}$ height, $45 \mathrm{~mm}$ diameter, $95 \mathrm{~g}$ Schunk GmbH \& Co. KG), firmly attached to the upper left leg just above the knee, with a comfortable surface on top (see Fig. 1). The sensor, which was calibrated statically, has been used previously in instrumented force shoes, and has been shown to measure force and center of pressure with errors below 1\% and $5 \mathrm{~mm}$, respectively (Faber et al., 2012; Schepers et al., 2007).

For each lift, subjects were instructed to walk towards the load, stand as close as comfortably possible to the load, and lift it with the instructed technique. Lifts started and ended with standing upright while holding the object with the hands at about hip level. The lifting techniques were (1) a free lifting technique, (2) a stoop technique, i.e. lifting by bending the back and keeping the knees extended, (3) a squat technique, i.e. lifting by bending the knees and rotating the knees
A

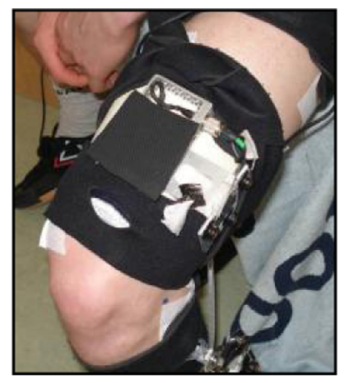

B
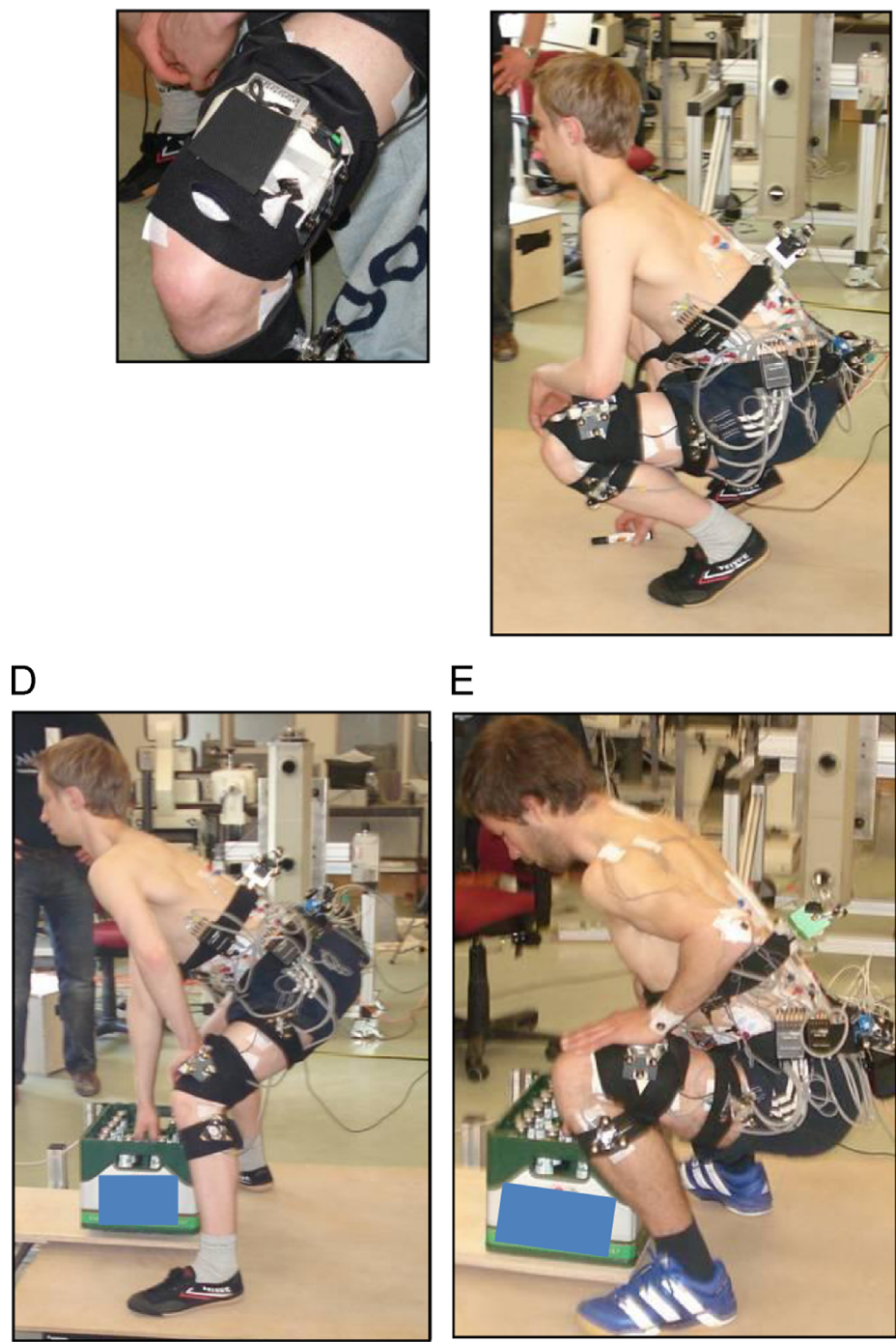

$\mathrm{E}$

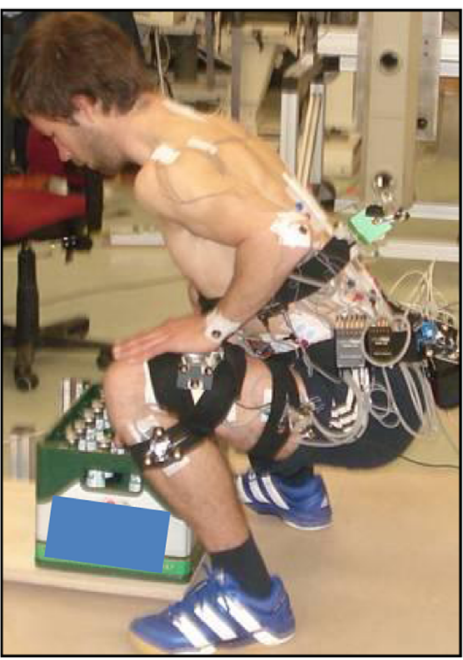

C

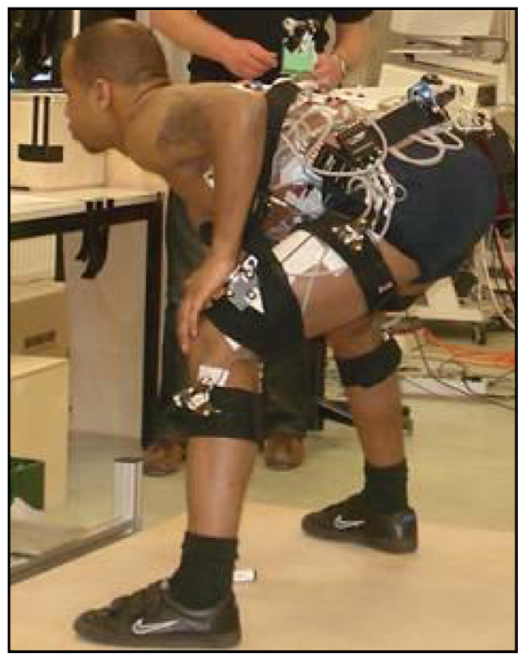

$\mathrm{F}$

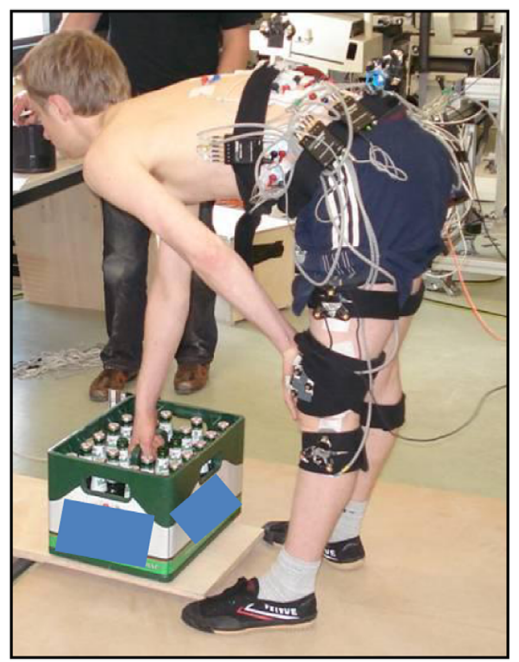

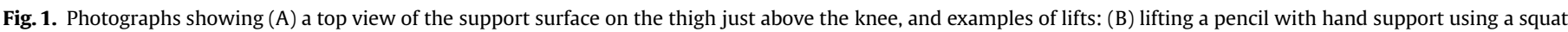

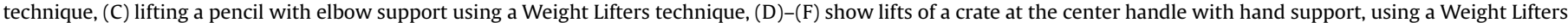
technique (D), a squat technique (E), and a stoop technique (F). 
outward while holding the trunk as upright as possible, (4) a weight lifters technique (WLT), which is characterized by a wide foot stance (lateral foot edges at $60 \%$ body height), moderately bend knees and flexed hips but a straight back. Lifting techniques were practiced before the experiment under supervision of a professional instructor.

\subsection{Measurements}

A single custom-made $1.0 \times 1.0 \mathrm{~m}$ force plate was used to measure ground reaction forces at 200 samples/s. Kinematics were collected at a sample rate of 50 samples/s using an opto-electronic automated 3D movement registration system (Optotrak, Norton Digital Inc.). LED cluster markers were attached to body segments (feet with lower legs, upper legs, pelvis and trunk) and to the force transducer. Prior to the measurements, for each participant, cluster markers were related to anatomical landmarks using pointer measurements (Cappozzo et al., 1995).

Fourteen pairs of surface EMG electrodes were attached to the trunk muscles (Rectus Abdominis, Internal Oblique, anterior and lateral part of External Oblique, Iliocostalis, and Longissimus pars thoracis and pars lumborum; see Kingma et al., 2010) after abrasion and cleaning with alcohol. EMG data were amplified (Porti17TM, TMS, Enschede, The Netherlands; input impedance $>10^{12} \Omega$, common mode rejection ratio $(C M R R)>90 \mathrm{~dB})$, band-pass filtered $(10-400 \mathrm{~Hz})$ and A-D converted (22 bits at $1000 \mathrm{~Hz}$ ) and stored synchronized to Optotrak and force plate data.

\subsection{Data analysis}

Marker data and force plate data were low-pass filtered using a bi-directiona 2nd order butterworth filter at a cut-off frequency of $5 \mathrm{~Hz}$. A dynamic bottom-up 3-D linked segment model (Kingma et al., 1996; anthropometry adapted according to Faber et al., 2011), adapted to include the force of the hand/elbow on the left thigh (See Appendix), was used to calculate net moments $\left(\mathbf{M}_{L 5 S 1}\right)$ and reaction forces at the L5S1 intervertebral disc Hof (1992), expressed in the pelvic coordinate system.

Off-line, EMG signals were full-wave rectified and low-pass filtered at $2.5 \mathrm{~Hz}$ (Potvin et al., 1996). EMG data were normalized to maximum voluntary contrac tions (McGill, 1991) and used as input to an EMG driven trunk muscle model. The model has been described in more detail previously (van Dieën, 1997; van Dieën and Kingma, 2005), and consisted of a compilation of anatomical data described by Stokes and Gardner-Morse (1995) for the back muscles and by McGill (1996) for the abdominal muscles. Briefly, the model consisted of 90 muscle slips crossing the L5S1 joint. For muscle slips crossing the L4 and T12 levels, nodes were used as points about which these long muscles were wrapped. In this way, the muscles follow the lumbar curvature during motion. Muscle forces were estimated as the product of the assumed muscle maximum stress, normalized EMG amplitude and correction factors for the instantaneous muscle length (Woittiez et al., 1984) and contraction velocity (van Zandwijk, 1998). For each participant, a best fit between net moments and muscle moments was obtained by optimizing, over all lifts performed by a participant, three values for each participant: the gain, i.e. a scaling factor between EMG amplitude and muscle stress, the position of the passive length-tension curve relative to the muscle optimum length, and a scaling factor for the passive length-tension curve. Finally, to obtain compression and shear forces at the L5S1 intervertebral joint, muscle forces and net reaction forces were summed after projecting them on the axes system connected to the L5S1 disc center. For convenience, shear forces pushing the upper vertebra (L5) forward were indicated as positive. Lumbar angles were obtained by Euler decomposition of thorax relative to the pelvic anatomical axes (order: flexion-extension, lateral bending, axial rotation).

\subsection{Statistics}

Outcome variables were peak support forces and support forces at the instant of peak extension moment, peak 3D net moment components and total moments, peak 3D lumbar angles and estimated peak 3D spinal forces. For each variable two repeated measures ANOVAs were performed with support condition (no support, elbow support and hand support for pencil lifting; two handed, one-handed and hand support for crate lifting) and lifting technique as within subject factors. Where applicable, support effects were further explored using Bonferroni post-hoc tests. A significance level of $p<0.05$ was used.

\section{Results}

The fit of the combined time series over all lifts between net total moments and EMG driven model total moments was acceptable, with correlations ranging from 0.81 to 0.94 , and root mean squared differences ranging from 13 to $22 \mathrm{Nm}$ (6-10\% of the highest average peak total moment) over subjects (Fig. 2).

\subsection{Pencil lifting}

For peak forces in the transducer (F_support_max, resultant of the three orthogonal force components) an interaction between hand condition and lifting technique $(p=0.048)$ was found (Table 1). For hand support, these forces ranged from $110 \mathrm{~N}$ $(\mathrm{SD}=38 \mathrm{~N})$ for stoop lifting to $179 \mathrm{~N}(\mathrm{SD}=59 \mathrm{~N})$ for free lifting. At the instant of the peak extension moment, the forces were substantially lower, with values of $39 \mathrm{~N}(\mathrm{SD}=50 \mathrm{~N}), 53 \mathrm{~N}(\mathrm{SD}=64 \mathrm{~N})$, $86 \mathrm{~N}(\mathrm{SD}=103 \mathrm{~N})$, and $121 \mathrm{~N}(\mathrm{SD}=76 \mathrm{~N})$, for stoop, squat, free and WLT lifts, respectively. The resultant reduction in peak total moment ranged on average from $17 \mathrm{Nm}(17 \%)$ in squat lifting to $35 \mathrm{Nm} \mathrm{(25 \% )}$ in the free lifting technique (Fig. 3), but the interaction of support condition with lifting technique was not significant (Table 1).

Peak support forces with elbow support were comparable to peak forces with hand support, ranging from $102 \mathrm{~N}(\mathrm{SD}=49 \mathrm{~N})$ in stoop lifting to $195 \mathrm{~N}(\mathrm{SD}=57 \mathrm{~N})$ in the free lifting technique. However, these forces peaks occurred early during lifting, and had dropped to below $12 \mathrm{~N}$ for all lifting techniques at the instant of the peak extension moment. Still, elbow support reduced peak total moments, with post hoc tests only reaching significance (on average $16 \mathrm{Nm}, 12 \%$ ) for the WLT.

Lumbar kinematics were affected to a minor extent by elbow and hand support in lifting a pencil (Fig. 4, Table 1). Peak lumbar flexion angle was somewhat increased by elbow support for WLT $\left(7^{\circ}\right)$ and squat lifting $\left(8^{\circ}\right)$ and to a lesser extent and not significantly, by hand support (Fig. 4). The peak lumbar lateral bending angle was only slightly reduced by elbow support in the WLT. The peak lumbar twist angle increased with elbow and hand support (Table 1), but post-hoc tests only showed significant increases (of about $4^{\circ}$ ) in the WLT.

Asymmetric moment components increased somewhat with support (Table 1), for lateral bending moment with up to $19 \mathrm{Nm}$ for elbow support in squat lifting, and for twist moment with up to $12 \mathrm{Nm}$ for hand support in the free lifting technique (Fig. 3).

Estimated resultant compression and forward shear forces were affected by support condition, and this was, just like the support forces, dependent on lifting technique (Table 1; Fig. 5). In stoop lifting, for both elbow and hand support, effects on estimated compression and forward shear forces were minor and mostly non-significant. In contrast, for the WLT, substantial effects (ranging from $21 \%$ to $28 \%$ ) were found for both elbow and hand support, for both compression and forward shear force. Lateral shear forces were small and unaffected by support condition.

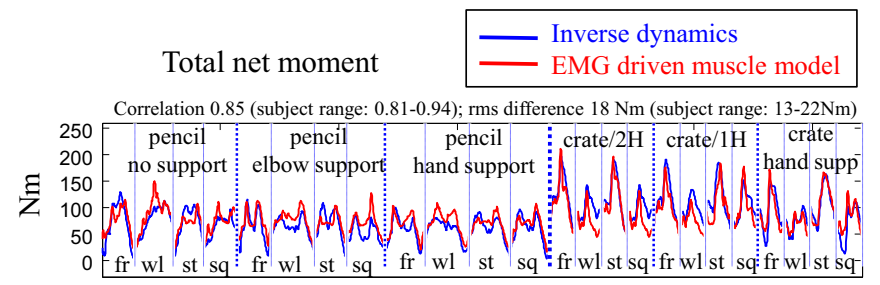

Fig. 2. Time series of total net moments from inverse dynamics and from the EMG drive muscle model for one representative subject. Time series for all condition are shown, with vertical lines separating time series between lifting techniques (thin solid lines; $\mathrm{fr}=$ free, $\mathrm{wl}=$ Weight Lifters Technique, $\mathrm{st}=$ stoop, $\mathrm{sq}=$ squat technique) and lifting conditions (dotted lines). Overall correlation and rms error are indicated above the figure. This specific subject showed some pronounced overestimations by the EMG driven model in pencil lifts. 
Table 1

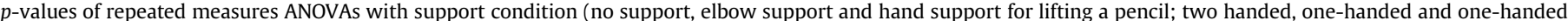

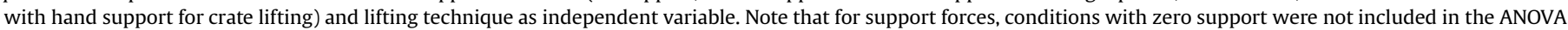

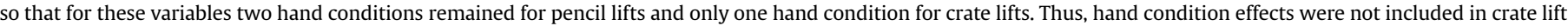
ANOVA's for support forces.

\begin{tabular}{|c|c|c|c|c|c|c|}
\hline & \multicolumn{2}{|l|}{ Support } & \multicolumn{2}{|c|}{ Technique } & \multicolumn{2}{|c|}{ Support * technique } \\
\hline & Pencil & Crate & Pencil & Crate & Pencil & Crate \\
\hline F_support_PEM ${ }^{\mathrm{a}}$ & .003 & - & .024 & .077 & .028 & - \\
\hline F_support_max & .180 & - & $<.001$ & $<.001$ & .048 & - \\
\hline Mnet_Extension ${ }^{c}$ & $<.001$ & $<.001$ & $<.001$ & $<.001$ & .167 & .008 \\
\hline Mnet_L5S1_Lateral & $<.001$ & $<.001$ & $<.001$ & .001 & .019 & $<.001$ \\
\hline Mnet_L5S1_twist & .002 & $<.001$ & $<.001$ & $<.001$ & .004 & .002 \\
\hline Mnet_L5S1_Total & $<.001$ & $<.001$ & $<.001$ & $<.001$ & .193 & .018 \\
\hline Angle_L5S1_Flexion $^{\mathrm{d}}$ & $<.001$ & .161 & $<.001$ & $<.001$ & .046 & .004 \\
\hline Angle_L5S1_Lateral & .143 & .005 & $<.001$ & .004 & .038 & .288 \\
\hline Angle_L5S1_twist & .016 & .002 & .101 & .026 & .083 & .726 \\
\hline F_L5S1_comp ${ }^{\mathrm{e}}$ & .002 & $<.001$ & .056 & $<.001$ & $<.001$ & .107 \\
\hline F_L5S1_shear ${ }^{\mathrm{e}}$ & $<.001$ & $<.001$ & $<.001$ & .112 & $<.001$ & $<.001$ \\
\hline F_L5S1_lat ${ }^{\mathrm{e}}$ & .469 & .001 & .124 & .588 & .414 & .508 \\
\hline
\end{tabular}

${ }^{a}$ F_support_PEM = total force (resultant of the three orthogonal force components) by the hand on the support surface at the instant of peak extension moment.

${ }^{b}$ F_support_max $=$ maximum total force (resultant of the three orthogonal force components) by the hand on the support surface.

${ }^{c}$ Mnet L5S1 = peak net moment at the L5S1 joint.

d Angle_L5S1 = peak lumbar angle.

e F_L5S1 = peak joint force at the L5S1 joint (comp=compression, shear=forward shear force, lat=lateral shear force).

\subsection{Crate lifting}

For crate lifting, peak support forces in the hand support condition ranged from $123 \mathrm{~N}(\mathrm{SD}=45 \mathrm{~N})$ in stoop lifting to $218 \mathrm{~N}$ $(\mathrm{SD}=65 \mathrm{~N})$ in the WLT. As with pencil lifting, support forces were substantially lower at the instant of the peak extension moment, ranging from $56 \mathrm{~N}(\mathrm{SD}=45 \mathrm{~N})$ for stoop lifting to $157 \mathrm{~N}(\mathrm{SD}=88 \mathrm{~N})$ for the free lifting technique. Compared to one-handed unsupported lifting, hand support resulted in a lifting techniquedependent reduction of peak total moments, ranging on average from $20 \mathrm{Nm}(13 \%)$ in squat lifting to $34 \mathrm{Nm}(19 \%)$ in free lifting (Table 1, Fig. 3). Compared to two-handed lifting, hand support reduced peak total moments, with reductions ranging from $20 \mathrm{Nm}$ (14\%) in squat lifting to $50 \mathrm{Nm}(26 \%)$ in free lifting. Peak lumbar flexion angle was somewhat affected by hand support, but this was not consistent across lifting techniques (Table 1, Fig. 4). Hand support slightly increased peak lateral bending $\left(2-5^{\circ}\right)$ and twisting angles $\left(3-4^{\circ}\right)$ relative to two-handed lifting. In contrast to unsupported two-handed and one-handed lifting, hand support resulted in most lifting techniques in substantial lateral bending moments (up to $54 \mathrm{Nm}, \mathrm{SD}=21 \mathrm{Nm}$, for the WLT) and twisting moments (up to $31 \mathrm{Nm}, \mathrm{SD}=10 \mathrm{Nm}$, for the WLT).

Hand support effects on estimated compression and shear forces ranged from non-significant for stoop lifting to a reduction of $17 \%$ (WLT) and $26 \%$ (free) for compression and $24 \%$ and $27 \%$ for forward shear (both for the WLT) when compared with unsupported one-handed lifting and two-handed lifting, respectively (Table 1, Fig. 5). Lateral shear forces were small but slightly increased with hand support in the WLT and squat lifts.

\section{Discussion}

The present study investigated the effect on low back loading of supporting the upper body by leaning on the thigh, for lifting a pencil and a crate. Compared to one-handed unsupported lifting, hand support resulted in a substantial reduction of peak total moments, both when lifting a pencil (range 17-25\% across lifting techniques) and when lifting a crate (range 13-19\% across lifting techniques). Compared to two-handed lifting of a crate, supported one-handed lifting had a somewhat more pronounced effect (range 14-26\%) on total moments, because, consistent with other studies (Arjmand et al., 2012; Ferguson et al., 2002; Kingma and van Dieën, 2004; Marras and Davis, 1998), two-handed lifting resulted in somewhat higher back loads than one-handed unsupported lifting.

In crate lifting, consistent with our hypothesis, hand support effects were dependent on lifting technique. In both stoop and squat lifting, support forces at the instant of peak moment were lower than in WLT and free lifts, which might be anticipated from inspection of Fig. 1. Accordingly, effects of hand support on peak total moments were also lower in stoop and squat lifts.

Elbow support, which was only used in pencil lifts, appeared to be much less effective than hand support, as subjects were unable to keep the elbow on the transducer over a substantial range of the lifting trajectory.

For compression and shear forces, we found hand support effects of a magnitude comparable to peak total moments, ranging from $13 \%$ to $28 \%$ except for stoop lifts, where hand support effects were not significant in both pencil and crate lifting.

Previous studies reported a 30\% total moment reduction (Kingma and van Dieën, 2004)and 15\% (Kingma and van Dieën, 2004) and a $16 \%$ (Ferguson et al., 2002) compression force reduction in onehanded lifting when leaning with the free hand on an external support. Comparison with the above-mentioned range of percentages in the present study suggests that leaning on the thigh is about as effective as leaning on an external object in terms of low back load reduction.

Although the effects of support were substantial, the question remains to what extent supporting the body by leaning on a thigh may help to reduce the risk of back injuries.

However, before interpreting absolute values of spine forces, potential sources of bias should be carefully considered. Errors in spinal forces estimated by our EMG-driven model may be due to factors such as cross-talk, i.e. capturing signals from neighboring muscles (DeLuca and Merletti, 1988), deep muscles being poorly represented in EMG signals (Stokes et al., 2003), EMG of wide muscles not optimally being captured with a single pair of electrodes (Staudenmann et al., 2005), EMG normalization and assumptions with regard to EMG-force relation. Furthermore, 

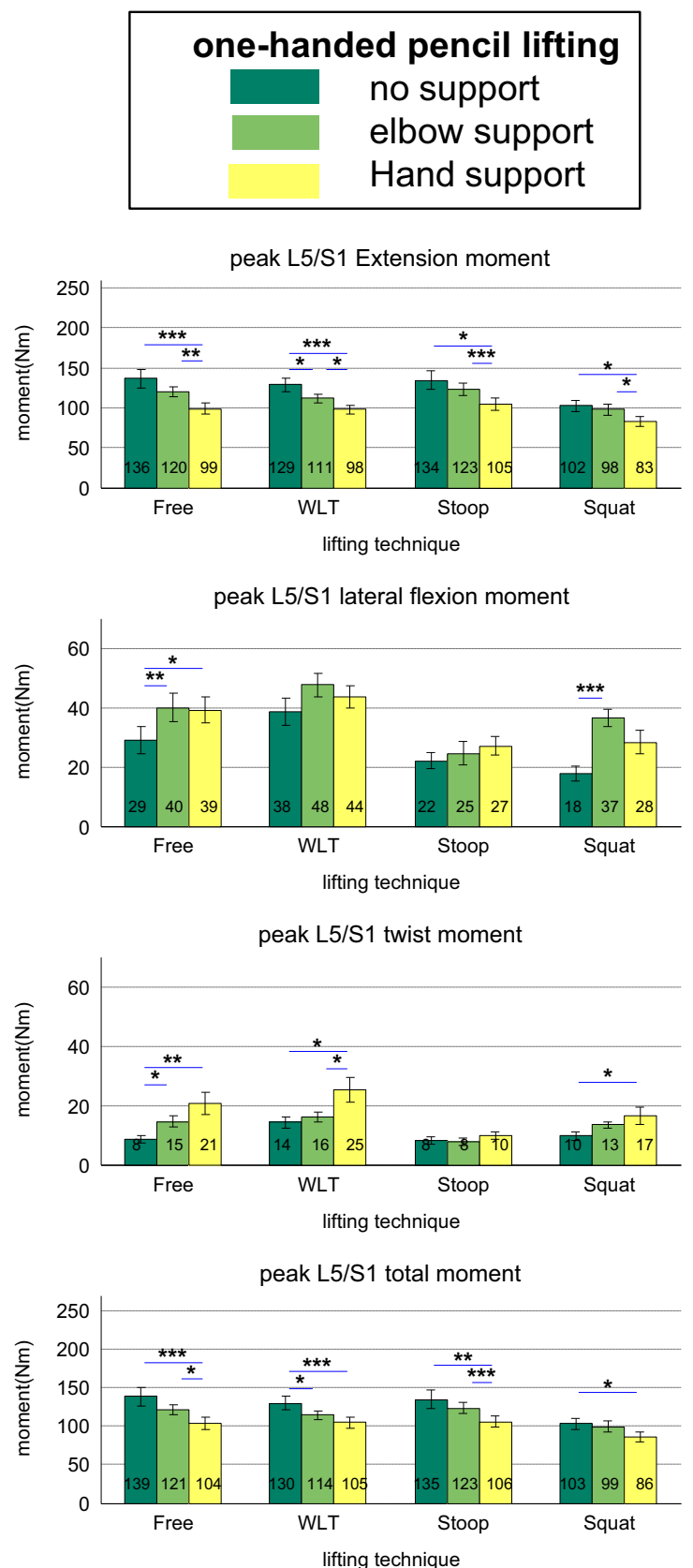
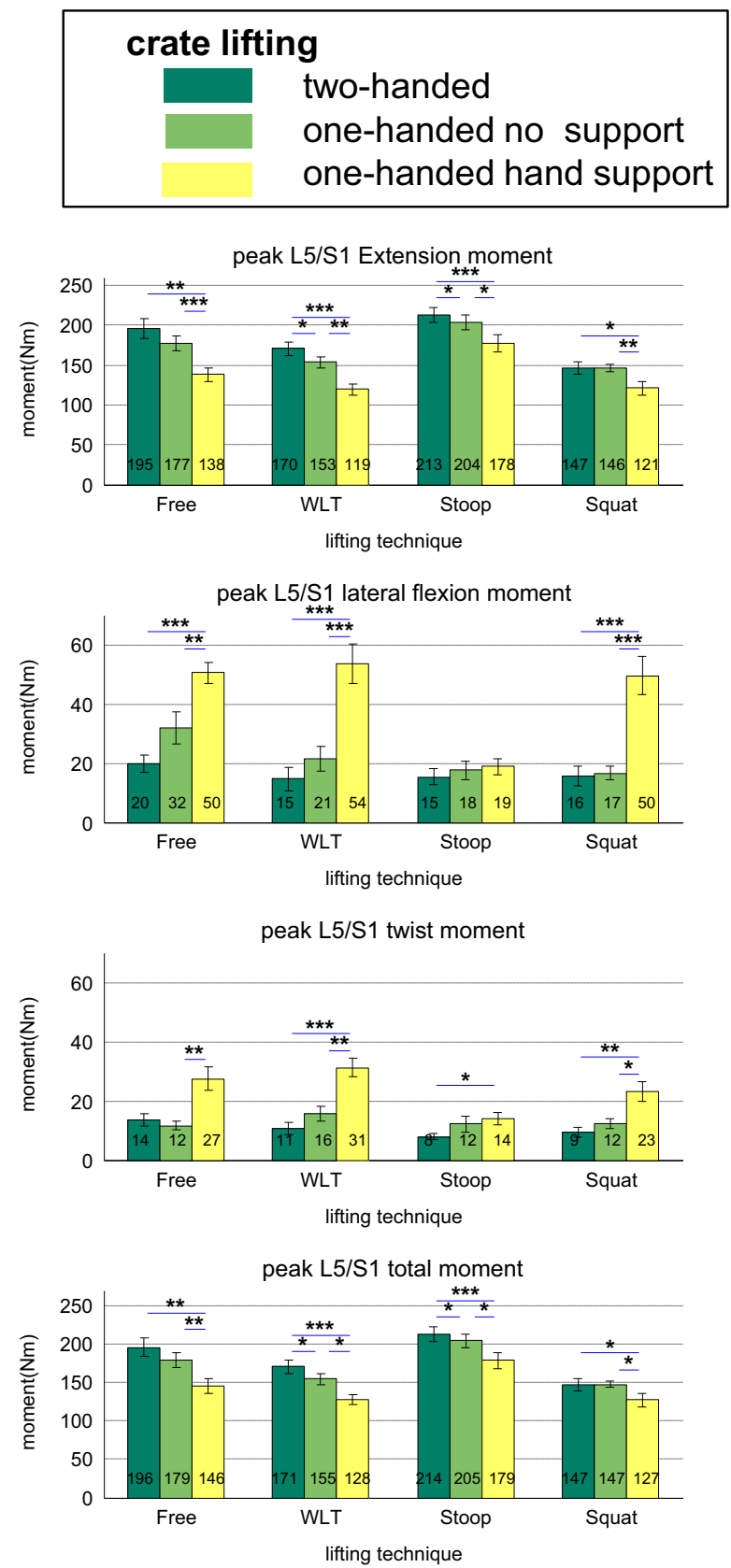

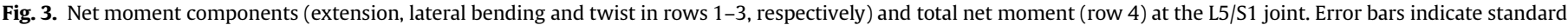

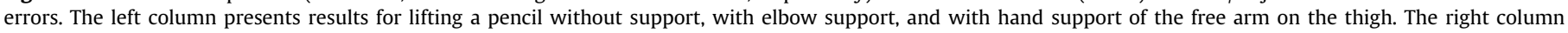

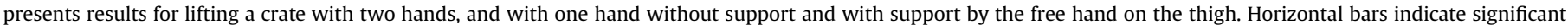
differences between pairs of condition according to bonferroni post-hoc tests. One, two and three stars indicate $p<0.05, p<0.01$ and $p<0.005$, respectively.

while our trunk model distributes motions over the lumbar spine, and incorporates wrapping of long muscles, simplifications such as ignoring translations in the spine, and considerations of moments at L5S1 only, may affect results (Arjmand et al., 2009; Gagnon et al., 2011).

However, differences in kinematics between hand conditions were small. Therefore, effects of the above-mentioned sources of error on our primary research question were probably small. Nevertheless, compression forces in some conditions require further consideration.

First, spine loads in pencil lifts, which ranged from 2600 to $3500 \mathrm{~N}$ across lifting techniques, may seem high when compared with previous findings for static full trunk flexion, ranging from
1532 to $2332 \mathrm{~N}$ across five estimation methods (Rajaee et al., 2015) and when compared to $1.6 \mathrm{MPa}$ measured in vivo (Wilke et al., 2001). However, it has been shown previously that dynamic lifting results in higher forces compared to static or slow lifts (e.g., de Looze et al., 1994; Kingma et al., 2001; Lavender et al., 1999). Indeed, Gagnon et al. (2011) reported peak L5S1 compression forces during unloaded dynamic full trunk flexion of 3107 when using a model requiring single-joint equilibrium and $3651 \mathrm{~N}$ when using a model requiring multijoint equilibrium. Bazrgari et al. (2008) calculated forces ranging from $2755 \mathrm{~N}$ for slow up to $4950 \mathrm{~N}$ for fast flexion/extension. All in all, compression forces in our study are within the range of expected values. 


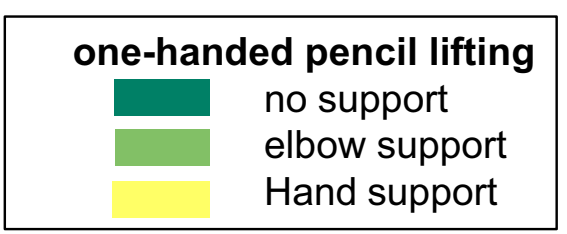

lumbar flexion angle
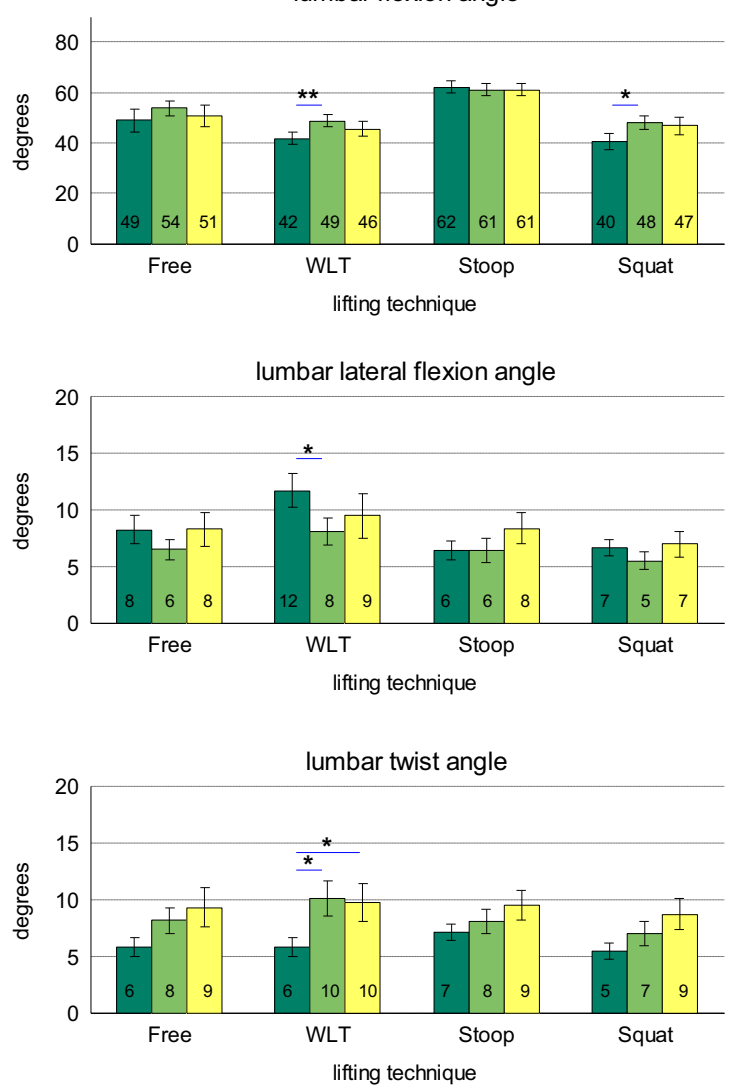

\section{crate lifting}

two-handed

one-handed no support one-handed hand support
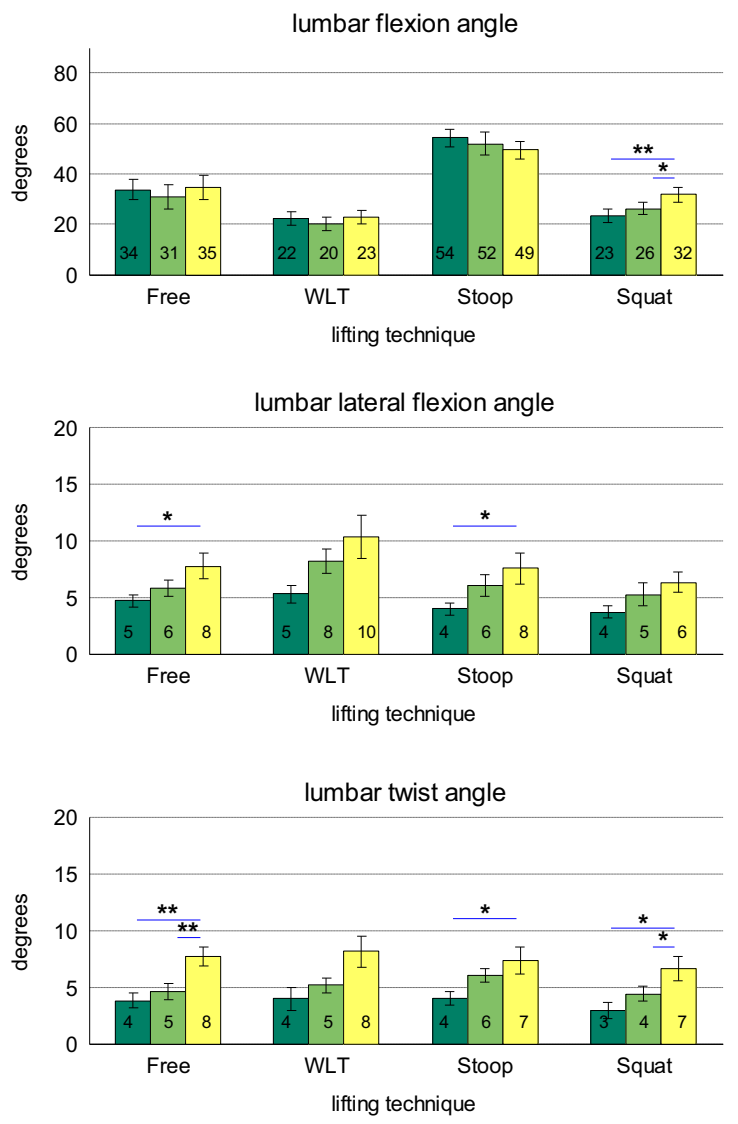

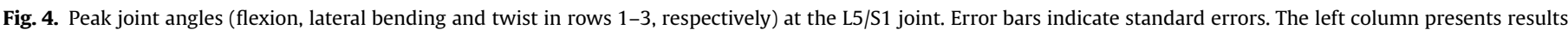

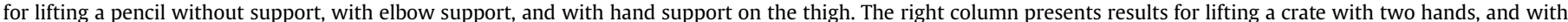

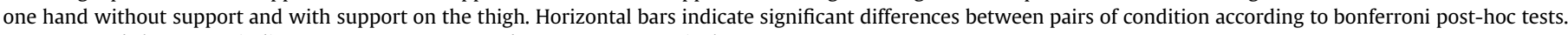
One, two and three stars indicate $p<0.05, p<0.01$ and $p<0.005$, respectively.

Furthermore, for some conditions, compression forces patterns deviated substantially from moments patterns. Specifically, in stoop lifts, hand support reduced net moments but not compression forces. The latter may be due to the difficulty in precisely predicting passive tissue forces in full flexion, as those forces depend on flexion in a highly non-linear way and are quite variable across subjects (Dolan and Adams, 1993). For the WLT, hand support effects were found in both pencil and crate lifts, but unexpectedly, compression forces were higher in pencil lifts than in crate lifts, whereas the opposite was the case for net moments. Indeed, for the WLT, the EMG driven model overestimated muscle moments (by on average $19 \mathrm{Nm}$ ) in pencil lifts and underestimated them (by on average $17 \mathrm{Nm}$ ) in crate lifts. This may be due to the limitations mentioned above. In addition, when reaching down to the floor, the WLT requires a combination of a straight back and strong hip flexion, which deviates from the natural pelvic-lumbar rhythm (Lariviere et al., 2000), and may have required high muscle forces to overcome passive tissue strains that are not adequately covered in the model. Indeed, back muscle EMG in the WLT was higher in pencil than in crate lifts.

For crate lifts, the present results suggest that two handed lifts can generate compression forces that would be high enough to cause damage to some spines when tested in vitro (Brinckmann, 1989), and that hand support may reduce this risk. For forward shear forces, the same line of reasoning holds, although for crate lifting with hand support forward shear forces were still higher than the recommended threshold of $1000 \mathrm{~N}$ (Gallagher and Marras, 2012). However, absolute shear force estimates should be treated even more cautiously than compression forces. While shear forces substantially above $1000 \mathrm{~N}$ have been reported before for comparable lifts (Bazrgari et al., 2007; Ferguson et al., 2002; Marras et al., 2003), others report lower values (Potvin et al., 1991; Rajaee et al., 2015). Shear force estimates are, more than compression force estimates, sensitive to modelling and postural details. Specifically, due to their sine relation with the shear axis, small changes in muscles lines of pull relative to disc orientation can have large effects on shear forces. This is also apparent when comparing shear force estimates across spine levels (Bazrgari et al., 2007; Gagnon et al., 2011; Kingma et al., 2004).

Reductions in total moments and spine loads due to hand support came at the expense of an increase in asymmetric moment components, which was minor in pencil lifts and substantial in crate lifts. The latter could, in combination with some (up to $5^{\circ}$ ) increase in asymmetric peak angles, result in co-contraction of abdominal muscles, 

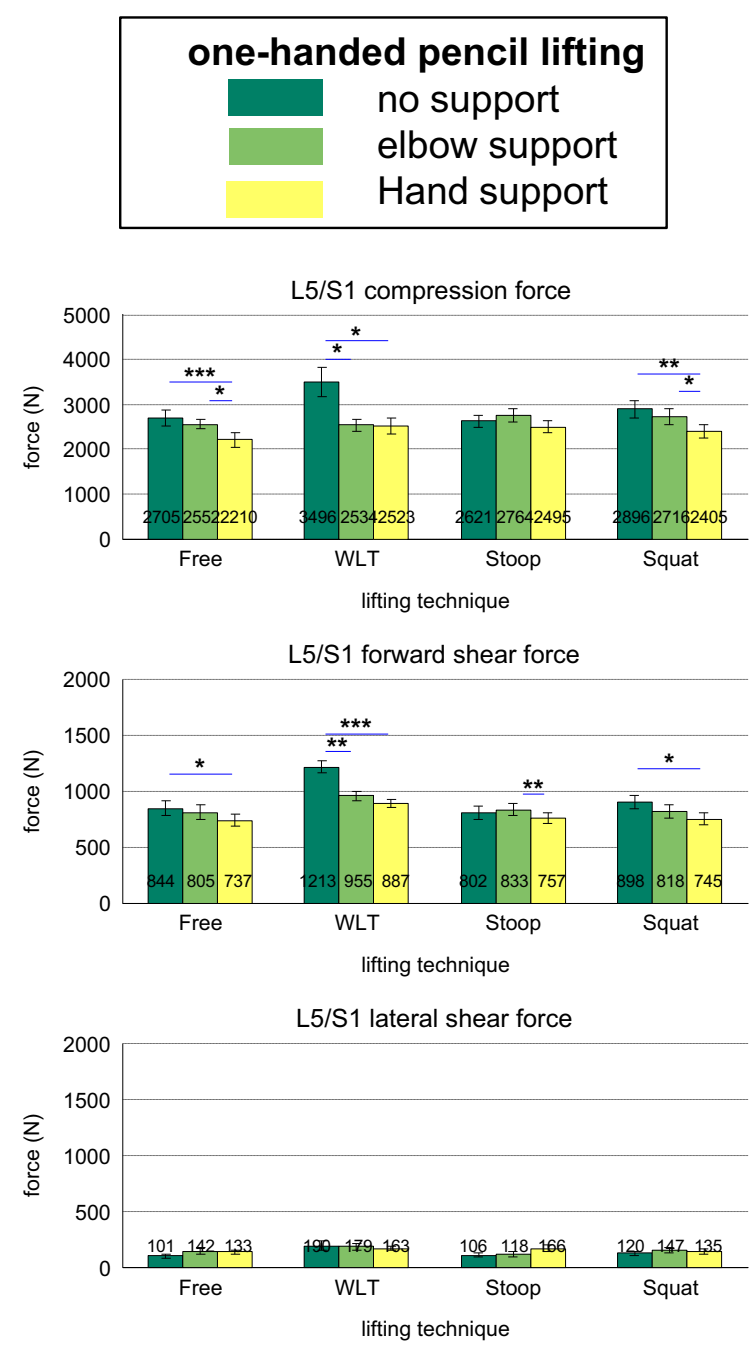
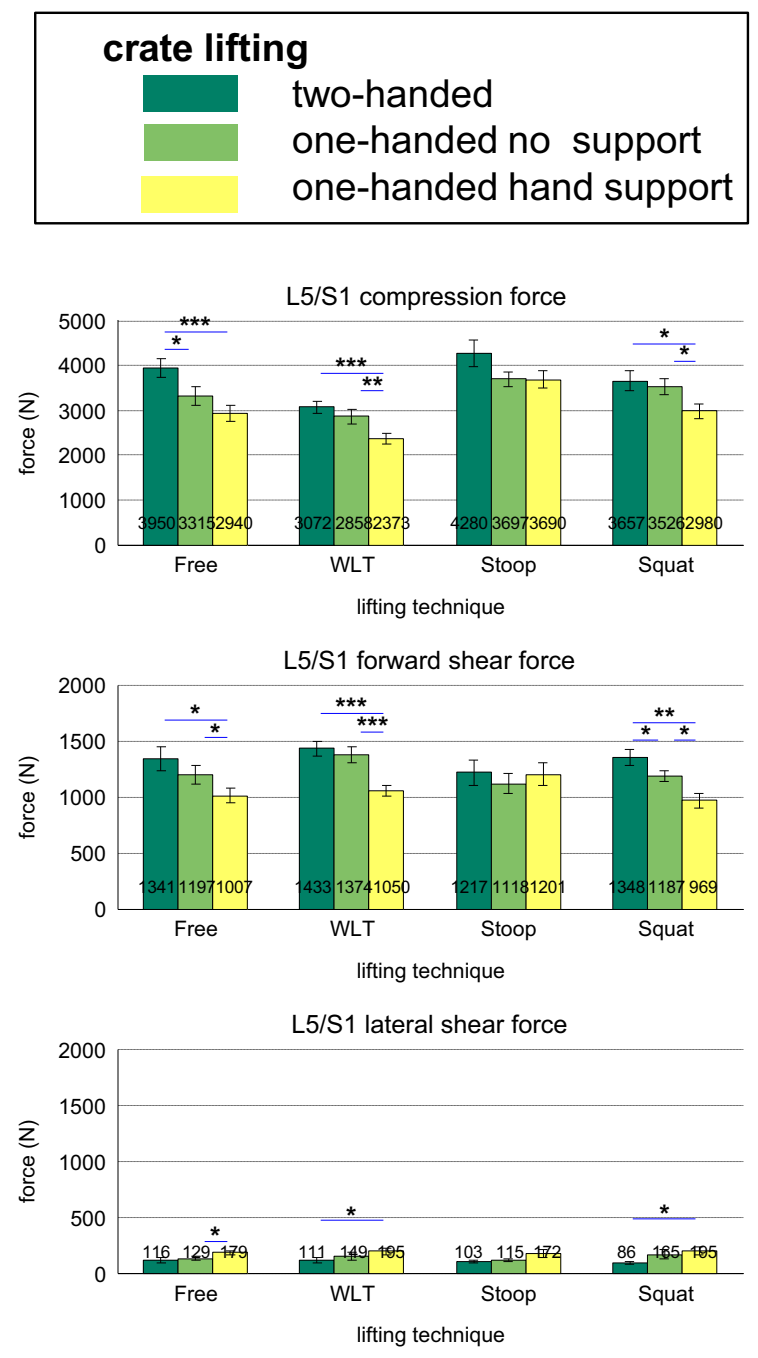

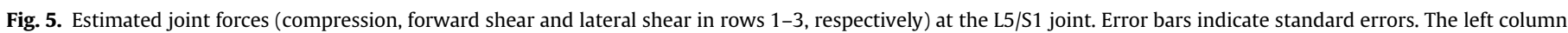

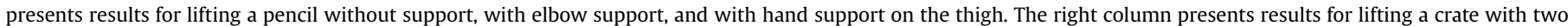

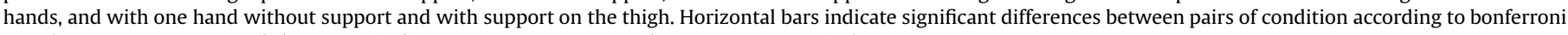
post-hoc tests. One, two and three stars indicate $p<0.05, p<0.01$ and $p<0.005$, respectively.

which increases compression, and in asymmetric spine loading. However, our estimated compression forces and lateral shear forces suggest that those effects were small. While trunk rotation has been suggested to be an independent risk factor for low back pain (Hoogendoorn et al., 2000), there is no evidence from the literature that the magnitude of asymmetry induced by hand support, is likely to cause such effects. Note also that even in symmetrical two-handed lifting, lateral bending moments of about $20 \mathrm{Nm}$ were found. While some influence of skin motion artifacts (Leardini et al., 2005) cannot be excluded here, lateral bending moment moments of this magnitude have been reported before for symmetrical lifts (Kingma et al., 2006; Lavender et al., 1999) and are plausible to occur in natural lifting conditions, as a lateral distance between the L5S1 joint and the center of pressure of the ground reaction force of about $2 \mathrm{~cm}$ is sufficient to cause such moments.

A limitation of this study is that the support surface on the thigh may feel differently from the natural situation where the subject really leans on the thigh, which may have affected results. Also, we may have slightly underestimated hand support effects, as some subjects may have touched the knee with the fingers despite the instruction to only lean with the palm of their hand on the support surface. Furthermore, as kinematics differed somewhat between hand conditions, effects of hand condition were a combined effect of hand force and actual lifting behavior rather than an effect of support force alone.

In addition, we did not address potential (long-term) adverse effects of one-handed lifting and hand support on knee and shoulder loading. Furthermore, our data showed substantial standard deviations across subjects in support forces and consequently in standard deviations of resulting effects of hand support on total net moments. In part, these variations may be due to differences in antropometric characteristics, such as body length and weight, as well as arm length and strength. Another limitation is that we only used male subjects. It has been shown previously that sex may interact with lifting technique (Lindbeck and Kjellberg, 2001; Marras et al., 2003).

Finally, it is obvious that hand support is only useful when objects can easily be lifted with one hand.

In conclusion, we found for lifting a pencil and for lifting a crate, hand support reduced total moments in all techniques, and estimated spinal forces were substantially reduced in all techniques except stoop lifts. When lifting a light object from the floor, hand support is more effective than elbow support. The down side of hand support was some increase of asymmetric motion and, for crate lifting, substantial asymmetric moments. 


\section{Conflict of interest statement}

There are no conflicts of interest.

\section{Acknowledgments}

The authors would like to thank STEP for partially financially supporting this project.

\section{Appendix A. Supplementary material}

Supplementary data associated with this article can be found in the online version at doi:10.1016/j.jbiomech.2015.09.035.

\section{References}

Anderson, C.K., Chaffin, D.B., 1986. A biomechanical evaluation of five lifting techniques. Appl. Ergon. 17, 2-8.

Arjmand, N., Gagnon, D., Plamondon, A., Shirazi-Adl, A., Lariviere, C., 2009. Comparison of trunk muscle forces and spinal loads estimated by two biomechanical models. Clin. Biomech. 24, 533-541.

Arjmand, N., Plamondon, A., Shirazi-Adl, A., Parnianpour, M., Lariviere, C., 2012. Predictive equations for lumbar spine loads in load-dependent asymmetric one- and two-handed lifting activities. Clin. Biomech. 27, 537-544.

Bazrgari, B., Shirazi-Adl, A., Arjmand, N., 2007. Analysis of squat and stoop dynamic liftings: muscle forces and internal spinal loads. Eur. Spine J. 16, 687-699.

Bazrgari, B., Shirazi-Adl, A., Trottier, M., Mathieu, P., 2008. Computation of trunk equilibrium and stability in free flexion-extension movements at different velocities. J. Biomech. 41, 412-421.

Brinckmann, P., 1989. Prediction of the compressive strength. Clin. Biomech. Suppl. $2,1-27$.

Brinckmann, P., Biggeman., M., Hilweg, D., 1989. Prediction of the compressive strength of human lumbar vertebrae. Spine 14, 606-610.

Burgess-Limerick, R., 2003. Squat, stoop or something in between? Int. J. Ind. Ergon. 31, 143-148.

Cappozzo, A., Catani, F., Croce, U.D., Leardini, A., 1995. Position and orientation in space of bones during movement: Anatomical frame definition and determination. Clin. Biomech. 10, 171-178.

Coenen, P., Kingma, I., Boot, C.R., Bongers, P.M., van Dieen, J.H., 2014. Cumulative mechanical low-back load at work is a determinant of low-back pain. Occup. Environ. Med. 71, 332-337.

Coenen, P., Kingma, I., Boot, C.R., Bongers, P.M., van Dieën, J.H., 2012. The contribution of load magnitude and number of load cycles to cumulative low-back load estimations: a study based on in-vitro compression data. Clin. Biomech. 27, 1083-1086.

de Looze, M.P., Dolan, P., Kingma, I., Baten, C.T.M., 1998. Does an asymmetric straddle-legged lifting movement reduce the low-back loading? Hum. Mov. Sci. 17, 243-259.

de Looze, M.P., Kingma, I., Thunissen, W., Wijk, M.J., van Toussaint, H.M., 1994. The evaluation of a practical model estimating lumbar moments in occupational activities. Ergonomics 37, 1495-1502.

DeLuca, C.J., Merletti, R., 1988. Surface myoelectic signal cross-talk among muscles of the leg. Electroencephalogr. Clin. Neurophysiol. 69, 568-575.

Dolan, P., Adams, M.A., 1993. Influence of lumbar and hip mobility on the bending stresses acting on the lumbar spine. Clin. Biomech. 8, 185-192.

Dolan, P., Earley, M., Adams, M.A., 1994. Bending and compressive stresses acting on the lumbar spine during lifting activities. J. Biomech. 27, 1237-1248.

Faber, G.S., Chang, C.C., Kingma, I., Martin Schepers, H., Herber, S., Veltink, P.H., Dennerlein, J.T., 2012. A force plate based method for the calibration of force/ torque sensors. J. Biomech. 45, 1332-1338.

Faber, G.S., Kingma, I., Bakker, A.J., van Dieën, J.H., 2009. Low-back loading in lifting two loads beside the body compared to lifting one load in front of the body. J. Biomech. 42, 35-41.

Faber, G.S., Kingma, I., van Dieen, J.H., 2011. Effect of initial horizontal object position on peak 15/s1 moments in manual lifting is dependent on task type and familiarity with alternative lifting strategies. Ergonomics 54, 72-81.

Ferguson, S.A., Gaudes-MacLaren, L.L., Marras, W.S., Waters, T.R., Davis, K.G., 2002. Spinal loading when lifting from industrial storage bins. Ergonomics 45, 399-414.

Gagnon, D., Arjmand, N., Plamondon, A., Shirazi-Adl, A., Lariviere, C., 2011. An improved multi-joint emg-assisted optimization approach to estimate joint and muscle forces in a musculoskeletal model of the lumbar spine. J. Biomech. 44, 1521-1529.

Gagnon, M., Plamondon, A., Gravel, D., 1993. Pivotting with the load. An alternative for protecting the back in asymmetric lifting. Spine 18, 1515-1524.

Gallagher, S., Marras, W.S., 2012. Tolerance of the lumbar spine to shear: a review and recommended exposure limits. Clin. Biomech. 27, 973-978.
Hof, A.L., 1992. An explicit expression for the moment in multibody systems. J Biomech, 25, 1209-1211.

Hoogendoorn, W.E., Bongers, P.M., de Vet, H.C., Douwes, M., Koes, B.W., Miedema, M.C., Ariens, G.A., Bouter, L.M., 2000. Flexion and rotation of the trunk and lifting at work are risk factors for low back pain: results of a prospective cohort study. Spine 25, 3087-3092.

Kingma, I., Baten, C.T., Dolan, P., Toussaint, H.M., van Dieen, J.H., de Looze, M.P. Adams, M.A., 2001. Lumbar loading during lifting: a comparative study of three measurement techniques. J. Electromyogr. Kinesiol. 11, 337-345.

Kingma, I., Bosch, T., Bruins, L., van Dieën, J.H., 2004. Foot positioning instruction, initial vertical load position and lifting technique: effects on low back loading. Ergonomics 47, 1365-1385.

Kingma, I., de Looze, M.P., Toussaint, H.M., Klijnsma, J.G., Bruijnen, T.B.M., 1996. Validation of a full body 3-d dynamic linked segment model. Hum. Mov. Sci. 15 833-860.

Kingma, I., Faber, G.S., Bakker, A.J., van Dieën, J.H., 2006. Can low back loading during lifting be reduced by placing one leg beside the object to be lifted? Phys. Ther. 86, 1091-1105.

Kingma, I., Faber, G.S., van Dieën, J.H., 2010. How to lift a box that is too large to fit between the knees. Ergonomics 53, 1228-1238.

Kingma, I., van Dieën, J.H., 2004. Lifting over an obstacle: effects of one-handed lifting and hand support on trunk kinematics and low back loading. J. Biomech. 37, 249-255.

Lariviere, C., Gagnon, D., Loisel, P., 2000. The effect of load on the coordination of the trunk for subjects with and without chronic low back pain during flexionextension and lateral bending tasks. Clin. Biomech. 15, 407-416.

Lariviere, C., Gagnon, D., Loisel, P., 2002. A biomechanical comparison of lifting techniques between subjects with and without chronic low back pain during freestyle lifting and lowering tasks. Clin. Biomech. 17, 89-98.

Lavender, S.A., Li, Y.C., Andersson, G.B., Natarajan, R.N., 1999. The effects of lifting speed on the peak external forward bending, lateral bending, and twisting spine moments. Ergonomics 42, 111-125.

Lavender, S.A., Li, Y.C., Natarajan, R.N., Andersson, G.B., 2009. Does the asymmetry multiplier in the 1991 niosh lifting equation adequately control the biomechanical loading of the spine? Ergonomics 52, 71-79.

Lavender, S.A., Lorenz, E.P., Andersson, G.B., 2007. Can a new behaviorally oriented training process to improve lifting technique prevent occupationally related back injuries due to lifting? Spine 32, 487-494.

Leardini, A., Chiari, L., Della Croce, U., Cappozzo, A., 2005. Human movement analysis using stereophotogrammetry. Part 3. Soft tissue artifact assessment and compensation. Gait Posture 21, 212-225.

Lindbeck, L., Kjellberg, K., 2001. Gender differences in lifting technique. Ergonomics 44, 202-214.

Marras, W.S., Davis, K.G., 1998. Spine loading during asymmetric lifting using one versus two hands. Ergonomics 41, 817-834.

Marras, W.S., Davis, K.G., Jorgensen, M., 2003. Gender influences on spine loads during complex lifting. Spine J. 3, 93-99.

Marras, W.S., Ferguson, S.A., Lavender, S.A., Splittstoesser, R.E., Yang, G., 2014 Cumulative spine loading and clinically meaningful declines in low-back function. Hum. Factors 56, 29-43.

McGill, S.M., 1991. Electromyographic activity of the abdominal and low back musculature during the generation of isometric and dynamic axial trunk torque: implications for lumbar mechanics. J. Orthop. Res. 9, 91-103.

McGill, S.M., 1996. A revised model of the abdominal musculature for torso flexion efforts. J. Biomech. 29, 973-977.

Norman, R., Wells, R., Neumann, P., Frank, J., Shannon, H., Kerr, M., 1998. A comparison of peak vs cumulative physical work exposure risk factors for the reporting of low back pain in the automotive industry. Clin. Biomech. 13, 561-573.

Potvin, J.R., Norman, R.W., McGill, S.M., 1996. Mechanically corrected emg for the continuous estimation of erector spinae muscle loading during repetitive lifting. Eur. J. Appl. Physiol. Occup. Physiol. 74, 119-132.

Potvin, J.R., Norman, R.W., McGill, S.M., 1991. Reduction in anterior shear forces on the 14/15 disc by lumbar musculature. Clin. Biomech. 6, 88-96.

Rajaee, M.A., Arjmand, N., Shirazi-Adl, A., Plamondon, A., Schmidt, H., 2015. Comparative Evaluation of Six quantitative lifting tools to estimate spine loads during static activities. Appl. Ergon., 48; , pp. 22-32.

Schepers, H.M., Koopman, H.F., Veltink, P.H., 2007. Ambulatory assessment of ankle and foot dynamics. IEEE Trans. Bio-med. Eng. 54, 895-902.

Staudenmann, D., Kingma, I., Stegeman, D.F., van Dieen, J.H., 2005. Towards optima multi-channel emg electrode configurations in muscle force estimation: a high density emg study. J. Electromyogr. Kinesiol. 15, 1-11.

Stokes, I.A., Henry, S.M., Single, R.M., 2003. Surface emg electrodes do not accurately record from lumbar multifidus muscles. Clin. Biomech. 18, 9-13.

Stokes, I.A.F., Gardner-Morse, M., 1995. Lumbar spine maximum efforts and muscle recruitment patterns predicted by a model with multijoint muscles and joints with stiffness. J. Biomech. 28, 173-186.

Straker, L.M., 2003. A review of research on techniques for lifting low-lying objects: 2. Evidence for a correct technique. Work 20, 83-96.

Troup, J.D.G., Leskinen, T.P.J., Stålhammar, H.R., Kuorinka, I.A.A., 1983. A comparison of intraabdominal pressure increases, hip torque, and lumbar intervertebral compression in different lifting techniques. Hum. Factors 25, 517-525.

van Dieën, J.H., 1997. Are recruitment patterns of the trunk musculature compatible with a synergy based on the maximization of endurance? J. Biomech. 30, 1095-1100.

van Dieën, J.H., Hoozemans, M.J.M., Toussaint, H.M., 1999. Stoop or squat: a review of biomechanical studies on lifting technique. Clin. Biomech. 14, 685-696. 
van Dieën, J.H., Kingma, I., 2005. Effects of antagonistic co-contraction on differences between electromyography based and optimization based estimates of spinal forces. Ergonomics 48, 411-426.

van Zandwijk, J.P., 1998. The Dynamics of Muscle Force Development. An Experimental and Simulation Study of the Behaviour of Human Skeletal Muscles (PhD thesis). VU University, Amsterdam.
Wilke, H., Neef, P., Hinz, B., Seidel, H., Claes, L., 2001. Intradiscal pressure together with anthropometric data-a data set for the validation of models. Clin. Biomech. 16 (Suppl. 1), S111-S126.

Woittiez, R.D., Huijing, P.A., Boom, H.K.B., Rozendal, R.H., 1984. A three-dimensional muscle model: a quantified relation between form and function of skeletal muscles. J. Morphol. 182, 95-113. 